\section{A CROSS-SECTIONAL STUDY OF PATIENTS WITH CHRONIC INFLAMMATORY DEMYELINATING POLYNEUROPATHY (CIDP): IDENTIFYING ULTRASONOGRAPHIC FEATURES FOR DIAGNOSIS AND PROGNOSIS}

1,2Nicholas Crump, ${ }^{1}$ Richard Macdonell, ${ }^{3}$ Michael Cartwright. ${ }^{1}$ Austin Health, Heidelberg, VIC, Australia; ${ }^{2}$ Departments of Medicine and Neurology, University of Melbourne, Austin Campus, Heidelberg, VIC, Australia; ${ }^{3}$ Department of Neurology, Wake Forest Baptist Medical Center, Winston-Salem, NC, USA

\subsection{6/bmjno-2021-ANZAN.25}

Objectives Diagnosis and treatment monitoring in CIDP is primarily based on clinical parameters. High-frequency ultrasound of peripheral nerves can reflect CIDP pathophysiology and changes with treatment. This project investigated potential diagnostic and prognostic biomarkers utilizing neuromuscular ultrasound.

Methods We conducted a standardized clinical and ultrasonographic assessment of 50 CIDP patients (25 at WFBMC, 25 at Austin), comparing to 25 healthy controls and 25 axonal neuropathy subjects. Our protocol included whole-length assessment of both median and ulnar nerves, with unilateral assessment of other nerves.

Results 25 of 25 CIDP patients studied at WFBMC had an abnormality on ultrasound (as determined by focal nerve enlargement determined by increased cross-sectional area), with 23 of 25 subjects having $>=4$ enlarged segments. 23 of 25 Austin CIDP patients had at least one enlarged segment, and 20 of 25 had $>=4$ enlarged segments. 46 of 48 had at least one abnormality in either median or ulnar nerve.

Mild nerve enlargements were infrequently seen in healthy and disease controls. However, CIDP patients had clear difference in extent and pattern of enlargements, particularly with proximal upper limb enlargement. Specific markers differentiating CIDP patients will be presented.

We analyzed our data in line with previously published diagnostic scores. We will discuss these findings for typical vs. atypical CIDP subtypes, and clinical correlations.

Conclusions This cross-sectional study of neuromuscular ultrasound in patients with CIDP suggests assessing bilateral wholelength median and ulnar nerves may be adequate for diagnosis, and differentiating potentially treatment responsive immune-mediated neuropathies from axonal neuropathies and healthy controls.

\section{POSTUROGRAPHY AS A BIOMARKER OF IVIG EFFICACY IN CIDP PATIENTS}

${ }^{1}$ Matthew Silsby, ${ }^{2}$ Con Yiannikas, ${ }^{2}$ Karl Ng, ${ }^{3,4}$ Matthew C Kiernan, ${ }^{1}$ Victor SC Fung, ${ }^{1}$ Steve Vucic. 'Neurology, Westmead Hospital, Westmead, NSW, Australia; ${ }^{2}$ Neurology, Royal North Shore Hospital, St Leonards, NSW, Australia; ${ }^{3}$ Neurology, Royal Prince Alfred Hospital, Sydney, NSW, Australia; ${ }^{4}$ Brain and Mind Centre, University of Sydney, Camperdown, NSW, Australia

\subsection{6/bmjno-2021-ANZAN.26}

Introduction Poor balance is a common and debilitating feature in patients with Chronic Inflammatory Demyelinating Polyradiculoneuropathy (CIDP). Posturography is an objective method of assessing balance. Intravenous Immunoglobulin (IVIG) exerts clinical benefits in CIDP, including improving balance, although this is difficult to quantify. The present study used posturography as a biomarker for determining IVIG efficacy in CIDP.
Methods Eighteen patients with CIDP established on IVIG were compared with healthy controls. Five conditions were used to assess balance: Feet $16 \mathrm{~cm}$ apart with eyes open and closed, feet together with eyes open and closed (Romberg's test), and tandem stance. Centre of pressure (COP) was sampled for 15 seconds at $100 \mathrm{~Hz}$ using a Kistler force platform, and the total path travelled by the COP was calculated (sway path). Testing was performed on the day of IVIG, corresponding to a trough, and at the mid-point of treatment, corresponding to a peak.

Results At baseline, there was a significant increase in the sway path in CIDP patients compared with healthy controls (average performance $1191 \pm 104 \mathrm{~mm}$ vs $724 \pm 26 \mathrm{~mm}$, $\mathrm{P}<0.001)$. Treatment with IVIG resulted in a significant reduction in the sway path when assessing Romberg's test (1759 \pm $324 \mathrm{~mm}$ vs $1081 \pm 134 \mathrm{~mm}, \mathrm{P}=0.019)$ and tandem stance $(1775 \pm 290 \mathrm{~mm}$ vs $1152 \pm 113 \mathrm{~mm}, \mathrm{P}=0.027)$. In contrast, clinical markers of neuropathy were unchanged, and repeat assessments in healthy controls were stable.

Conclusion The present study shows that objective assessment of balance using posturography may serve as a biomarker of IVIG efficacy in patients with CIDP.

\section{EFFECT OF INTER-HOSPITAL TRANSFER IN PATIENTS UNDERGOING ENDOVASCULAR THROMBECTOMY IN THE EARLY AND LATE TIME WINDOW}

1,2,3 Leon Edwards, 1,2,3 Christopher Blair, 1,2,3 Dennis Cordato, 4,5,6 Nathan Manning 2,3,4,5 Andrew Cheung, ${ }^{4,5,7}$ Jason Wenderoth, ${ }^{1,2,3}$ Cecilia Cappelen-Smith. ${ }^{1}$ Neurology and Neurophysiology, Liverpool Hospital, Liverpool, NSW, Australia; 'Ingham Institute for Applied Medical Research, Ingham Institute, Liverpool, NSW, Australia; ${ }^{3}$ South Western Sydney Clinical school, University of New South Wales, Liverpool, NSW, Australia; ${ }^{4}$ Institute of neurological sciences, Prince of Wales Hospital, Randwick, NSW, Australia; ${ }^{5}$ Neurointervention, Liverpool Hospital, Liverpool, NSW, Australia; ${ }^{6}$ The Florey institute of neurosciences, Melbourne, VIC, Australia; ${ }^{7}$ Prince of Wales Clinical School, Prince of Wales, Randwick, NSW, Australia

\subsection{6/bmjno-2021-ANZAN.27}

Objective Assess the effect of inter-hospital transfer and treatment time on mortality, reperfusion rates and workflow time metrics in patients undergoing endovascular thrombectomy (EVT) for acute ischaemic stroke (AIS) due to large vessel occlusion (LVO) in the anterior cerebral circulation (ACC).

Methods Analysis of a prospective database of consecutive patients undergoing EVT for LVO presenting between January 2017-December 2018 at a single Australian comprehensive stroke centre (CSC). Patients presented directly or were transferred to the CSC from 21 sites across New South Wales and the Australian Capital Territory. A definition of $\leq 6$ hours or $>6$ hours of stroke onset to treatment (groin puncture) was used for the early time window (ETW) and late time window (LTW) respectively.

Results 154/213 (72\%) patients were inter-hospital transfers. There was no significant difference in baseline characteristics including age, National-Institute-of-Health-Stroke-Scale-score, intravenous thrombolysis administration or procedure time between transferred and direct presenters (all $\mathrm{p}>0.05$ ). Transferred patients within the ETW had worse 90-day functional outcome $(35.6 \%$ vs $61.0 \%$, Odds ratio [OR] 0.36 , 95\% confidence interval [CI] 0.17-0.75), higher mortality $(25.3 \%$ vs $6.8 \%$, OR 6.57, CI 1.48-29.32) and longer stroke-onset to treatment time $(180$ vs 245 minutes, $\mathrm{p}<0.01)$. In the LTW transferred patients there was no significant difference in 90 - 
day functional outcome, mortality or stroke-onset to treatment compared with the directly presenting patients (all p>0.05). Successful reperfusion rates and $\mathrm{sICH}$ were similar between cohorts (all p>0.05)

Conclusion Inter-hospital transfer in the ETW but not LTW is associated with longer stroke-onset to treatment, worse 90-day functional outcome and higher mortality.

\section{ADJUNCTIVE INTRAARTERIAL THROMBOLYSIS IN ENDOVASCULAR CLOT RETRIEVAL: A SYSTEMATIC REVIEW AND META-ANALYSIS}

${ }^{1}$ William K Diprose, ${ }^{2}$ Michael TMTM Wang, ${ }^{1}$ Kaustubha Ghate, ${ }^{3}$ Stefan Brew, ${ }^{3}$ James R Caldwell, ${ }^{3}$ Ben McGuinness, ${ }^{2} \mathrm{P}$ Alan Barber. 'Department of Neurology, Auckland City Hospital, Auckland, New Zealand; 'Department of Medicine, University of Auckland, Auckland, New Zealand; ' ${ }^{3}$ Department of Radiology, Auckland City Hospital, Auckland, New Zealand

\subsection{6/bmjno-2021-ANZAN.28}

Objective To evaluate the safety and efficacy of intra-arterial thrombolysis (IAT) as an adjunct to endovascular clot retrieval (ECR) in ischaemic stroke, we performed a systematic review and meta-analysis of the literature.

Methods Searches were performed using Medline, Embase, and Cochrane databases for studies that compared ECR to ECR with adjunctive IAT (ECR+IAT). Safety outcomes included symptomatic intracerebral haemorrhage (sICH) and mortality at three months. Efficacy outcomes included successful reperfusion (Thrombolysis in Cerebral Infarction score of $2 \mathrm{~b}$ to 3 ), and functional independence, defined as a modified Rankin Scale score of 0 to 2 at three months.

Results Five studies were identified that compared combined ECR+IAT (IA alteplase or urokinase) to ECR-only, and were included in the random effects meta-analysis. There were 1693 ECR patients, including 269 patients treated with combined ECR+IAT and 1424 patients receiving ECR-only. Pooled analysis did not demonstrate any differences between ECR +IAT and ECR-only in rates of sICH (OR: 0.61, 95\% CI: $0.20-1.85 ; \mathrm{P}=0.78$ ), mortality (OR: $0.77,95 \% \mathrm{CI}: 0.54-1.10$; $\mathrm{P}=0.15$ ), or successful reperfusion (OR: 1.05 , 95\% CI: $0.52-$ $2.15 ; \mathrm{P}=0.89)$. There was a higher rate of functional independence in patients treated with ECR+IAT, although this was not statistically significant (OR: 1.34, 95\% CI: 1.00-1.80; $\mathrm{P}=0.053)$.

Conclusions Adjunctive IAT appears to be safe. In specific situations, neurointerventionists may be justified in administering small doses of intraarterial alteplase or urokinase as rescue therapy during ECR.

\section{HIGH SENSITIVITY TROPONIN IN ACUTE ISCHAEMIC STROKE STUDY (TACIS)}

${ }^{1}$ Andrew Hannaford, ${ }^{2}$ Michael Hayes, ${ }^{3}$ John Worthington, ${ }^{3}$ Timothy Ang, ${ }^{2}$ Nimalin Harinesan. 'Neurology, Westmead Hospital, Sydney, NSW, Australia; ${ }^{2}$ Neurology, Concord Repatriation General Hospital, Sydney, NSW, Australia; ${ }^{3}$ Neurology, Royal Prince Alfred Hospital, Sydney, NSW, Australia

\subsection{6/bmino-2021-ANZAN.29}

Objective We designed a multi-centre prospective cohort study to explore the hypothesis that early acutely elevated high sensitivity troponin (hsT) is associated with cardioembolic stroke (CES)
Methods Ischaemic strokes across three hospitals underwent hsTroponin testing and 2 blinded clinicians classified patients as CES, NCE (Non Cardioembolic) or ESUS by ESUS criteria. Characteristics included baseline NIHSS, renal function, hypertension, diabetes, smoking, ischaemic heart disease, past stroke and congestive cardiac failure. The odds of positive hsT for CES Vs NCE and ESUS Vs NCE were modelled with stepwise addition of patient characteristics.

Results 194 ischaemic stroke cases were included, with a mean age of 71 years and a 57:43 male:female ratio. 65 had a positive hsTroponin, which was associated with older age, hypertension, cardiac failure, coronary disease, an eGFR $<60$ and a higher NIHSS. Positive hsTroponin was associated with CES (OR, 2.06; 95\% CI, 1.12-3.79; $\mathrm{P}=0.02$ ). This association persisted after adjusting for confounders, such as age, sex, atrial fibrillation, renal impairment, ischaemic heart disease and previous stroke (Adjusted OR, 4.07; 95\% CI, 1.41-11.75; $\mathrm{P}=0.01)$. ESUS was negatively associated with an elevated hs troponin (OR, 0.45; 95\% CI, 0.22-0.94; $P=0.03$ ). This was not significant when adjusting for other variables $(\mathrm{P}=0.09)$.

Conclusions An elevated hs troponin after acute ischaemic stroke is independently associated with a cardioembolic mechanism. High sensitivity cardiac troponin was not significantly associated with ESUS after adjusting for confounders, suggesting that a cardio-embolic cause may not be the dominant mechanism in this group.

\section{BRAIN AGING AND CARDIOVASCULAR RISK FACTORS IN CHRONIC HIV: A LONGITUDINAL MRI STUDY}

\begin{abstract}
1,2,3 David Jakabek, ${ }^{2,3}$ Caroline D Rae, 1,2,3,4Bruce J Brew, 1,2,5 Lucette A Cysique. ${ }^{1}$ Departments of Neurology and HIV Medicine, St Vincent's Hospital, and Peter Duncan Neurosciences Unit, St Vincent's Centre for Applied Medical Research, Sydney, NSW, Australia; ${ }^{2}$ Neuroscience Research Australia, Sydney, NSW, Australia; ${ }^{3}$ Faculty of Medicine, University of New South Wales, Sydney, NSW, Australia; ${ }^{4}$ Faculty of Medicine, University of Notre Dame, Sydney, NSW, Australia; ${ }^{5}$ UNSW Psychology, University of New South Wales, Sydney, NSW, Australia
\end{abstract}

\subsection{6/bmino-2021-ANZAN.30}

Objectives We aimed to examine the relative contributions of HIV infection, age, and cardiovascular risk factors to subcortical brain atrophy.

Methods Virally suppressed HIV+ participants with low neuropsychological confounds $(\mathrm{n}=75)$ and demographically matched HIV- controls ( $\mathrm{n}=31$ ) completed baseline and 18month follow-up MRI scans, neuropsychological evaluation, cardiovascular assessments, and laboratory tests. HIV + participants were evaluated for HIV associated neurocognitive disorder (HAND). Subcortical volumes were extracted with Freesurfer. Volumetric and shape analyses were conducted using linear mixed-effect models incorporating interactions between age, time, and each of HIV status, HAND status, HIV disease factors, and cardiovascular markers.

Results HIV+ participants had smaller volumes of most structures compared to HIV- participants. Premature aging was evident in the pallidum using volumetric $(p=0.032)$ and shape analyses. Accelerated aging was observed in the caudate volumes for the more severe HAND subgroup $(\mathrm{p}=0.008)$ and was associated with longer HIV duration for putamen volumes $(\mathrm{p}=0.04)$. Higher CD4 counts had a protective effect on hippocampal volumes in older participants $(p=0.04)$. Cardiovascular measures were associated with smaller volumes across time for most structures; only the putamen demonstrated 